\title{
Le partenariat comme déclencheur de la recherche participative dans un projet école-musée-famille pour/par le plurilinguisme
}

Raquel Carinhas, Maria Helena Araújo e Sá et Danièle Moore

\section{(2) OpenEdition}

Édition électronique

URL : https://journals.openedition.org/rdlc/8112

DOI : $10.4000 /$ rdlc. 8112

ISSN : 1958-5772

Éditeur

ACEDLE

Référence électronique

Raquel Carinhas, Maria Helena Araújo e Sá et Danièle Moore, « Le partenariat comme déclencheur de la recherche participative dans un projet école-musée-famille pour/par le plurilinguisme », Recherches en didactique des langues et des cultures [En ligne], 17-2 | 2020, mis en ligne le 27 avril 2020, consulté le 17 octobre 2022. URL : http://journals.openedition.org/rdlc/8112 ; DOI : https://doi.org/10.4000/rdlc. 8112

Ce document a été généré automatiquement le 17 octobre 2022.

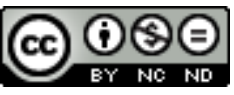

Creative Commons - Attribution - Pas d'Utilisation Commerciale - Pas de Modification 4.0 International - CC BY-NC-ND 4.0

https://creativecommons.org/licenses/by-nc-nd/4.0/ 


\title{
Le partenariat comme déclencheur de la recherche participative dans un projet école-musée-famille pour/ par le plurilinguisme
}

\author{
Raquel Carinhas, Maria Helena Araújo e Sá et Danièle Moore
}

\section{Introduction}

1 Dans les écologies plurilingues qui forment le tissu de nos sociétés et de l'école, de nombreux chercheurs soulignent à la fois l'importance de construire la continuité entre les différents espaces où circulent et apprennent les enfants (Ishimaru et al., 2015 ; Simonin \& Thamin, 2018), et l'importance pour les enseignants de travailler en étroite collaboration avec les familles et les communautés pour construire le projet pédagogique qui sera le leur. Il existe encore peu de recherches didactiques qui permettent d'explorer finement comment les pratiques linguistiques et culturelles et les savoirs des familles, des communautés locales et des institutions, peuvent être investis dans les partenariats éducatifs (Bottoms, Ciechanowski, Jones, de la Hoz, \& Fonseca, 2017) ni comment une recherche participative, fondée sur la collaboration, la réflexivité et le partage des expertises, se déploie sur le plan méthodologique et conceptuel.

2 La recherche en éducation et en didactique des langues et du plurilinguisme (DLP) a pourtant largement montré, depuis plusieurs décennies, que des projets partenariaux entre école, familles et communautés contribuent au succès scolaire et au développement intégral des élèves (Ishimaru, Barajas-López, \& Bang, 2015 ; Santos, Araújo e Sá, \& Simões, 2012), tout en soulignant l'importance de créer des situations éducatives œuvrant pour la mise en continuité des espaces de socialisation et d'apprentissage des apprenants (Simonin \& Thamin, 2018). Toutefois, peu de recherches se centrent sur les partenariats pédagogiques multi-situés, impliquant tout 
à la fois l'école, les musées et les familles, notamment autour de projets centrés sur le plurilinguisme (Moore, sous presse). Encore moins d'études se centrent plus particulièrement sur les dynamiques réflexives de mutualisation des expertises des réseaux d'acteurs-chercheurs dans le développement collaboratif de ressources pédagogiques plurilingues (Beaumont \& Moore, 2020).

3 C'est dans ce creux que s'inscrit notre réflexion qui porte sur l'analyse d'un partenariat éducatif autour du plurilinguisme comme déclencheur d'approches participatives (Anadon, 2013), dans le sens où celles-ci supposent aussi la coopération et la contribution des partenaires à la démarche d'investigation sur un même objet de recherche (Desgagné, 1997). Après une revue de littérature sur les défis des projets partenariaux développés sur la diversité linguistique et culturelle en DLP, nous ${ }^{1}$ présentons notre étude qui s'inscrit dans une perspective auto et ethnographique pour mieux comprendre comment se développe, du point de vue des acteurs, ce processus partenarial visant l'éducation au plurilinguisme d'enfants de l'école primaire en Uruguay.

\section{Contexte de la recherche et méthodologie}

4 Cette contribution se situe dans le contexte de l'éducation primaire en Uruguay, niveau d'enseignement qui s'adresse à des élèves de 6 à 12 ans. L'école de notre étude est une école de Tiempo Completo $^{2}$ (temps complet), située dans un quartier défavorisé de la ville de Montevideo. Cette école présente le pourcentage le plus élevé d'élèves étrangers (issus majoritairement de l'Amérique Latine) en Uruguay. La langue de scolarisation est l'espagnol et les enfants apprennent l'anglais et le portugais comme langues étrangères. Même si les politiques linguistiques du pays soulignent l'importance d'un curriculum en langues selon une orientation plurilingue, les programmes invitent plutôt à juxtaposer les apprentissages des différentes langues enseignées.

5 Le projet de partenariat en étude implique l'école en question (notamment sa directrice, des enseignants, des enfants et leurs familles), les trois chercheuses, et plusieurs musées de la ville : le MUMI - Museo de las Migraciones, le MAPI - Museo de Arte Precolombino e Indígena, et le Museo Histórico Cabildo). Au moment de la recherche (2019), seul le Musée des Migrations proposait déjà des ateliers adressés à des enfants avec une approche interculturelle. Pour les autres institutions muséales du réseau, le plurilinguisme restait encore une voie à explorer.

6 Ce partenariat a conçu un projet visant une approche plurilingue qui a été développé pendant une année scolaire (de mars à novembre de 2019). La recherche, de type ethnographique, a permis le recueil de plusieurs types de données: des observations participantes lors des activités (de la planification à la mise en œuvre à l'école et dans les musées), des enregistrements des interactions synchrones et asynchrones entre les partenaires (pendant la planification et les phases de rétroaction) et le journal réflexif et le carnet de recherche de la chercheuse principale (note 1). 


\section{Plurilinguismes et partenariats éducatifs école, familles et institutions muséales}

7 Peu de recherches ont été menées en contexte partenarial mobilisant les institutions muséales (Moore, Hoskyn, \& Mayo, 2018), malgré leur potentiel reconnu pour développer des programmes sur la diversité culturelle et linguistique (Charalampopoulou, 2013). Ce sont en effet des lieux d'apprentissage et facilitateurs de rencontres interculturelles (Walton, Paradies, \& Mansouri, 2016). Un état des lieux de ces recherches montre l'ampleur des défis relevés par le partage d'expertise entre praticiens, chercheurs et partenaires éducatifs lorsqu'ils mettent en place des projets éducatifs qui prennent en compte la diversité des langues et des cultures des apprenants. La complémentarité des expertises et des cultures de travail est vécue par les partenaires à la fois comme une potentialité et comme une contrainte. En effet, si cette complémentarité rend possible le développement de projets éducatifs perçus comme cohérents entre les contextes formels et non-formels d'apprentissage, elle implique aussi des tensions fréquentes entre les divers acteurs éducatifs. La dynamique partenariale demande donc qu'il soit possible de gérer ces tensions d'une façon productive à l'égard des objectifs du partenariat (Auger, Azaoui, Houée, \& Miquel, 2018). Le public cible de la plupart des recherches recensées sont des populations minorisées, notamment des allophones (Clerc, 2015), des immigrés (Bautista GarciaVera, Limón Mendizábal, Oñate, De La Rasilla, \& Rostand Quijada, 2016), des Roms (Auger et al., 2018) ou bien des autochtones (Flückiger, Diamond, \& Jones, 2012). Dans ce cadre, et quand ils incluent la participation des parents (surtout des mères, voir par exemple Auger et al., 2018 ; Flückiger et al., 2012), les projets pédagogiques sur lesquels portent ces études visent pour la plupart la prise de voix des populations minorisées et, par conséquent, la valorisation des langues minoritaires, des langues de la maison et des répertoires plurilingues des enfants (Auger et al., 2018 ; Flückiger et al., 2012 ; Li, Anderson, Carr, \& Hare, 2018).

8 Pour ce qui concerne les dynamiques des projets conduits à travers la participation de différentes institutions ayant des responsabilités éducatives, les études montrent qu'elles ne sont pas uniformes et exigent la constante adaptation aux contextes et aux cultures des plusieurs partenaires (Beaumont \& Moore, 2020 ; Clerc, 2015 ; Milutinović \& Gajić, 2010).

9 Le degré de participation des partenaires est flexible et instable, chaque partenaire pouvant assumer différents rôles tout au long d'un même projet. Dans les projets partenariaux impliquant une collaboration entre école et familles, la participation de ces dernières reste plutôt périphérique au projet d'école, et relève plus généralement du volontariat et des apprentissages à la maison (Epstein, 2002)3. Mais lorsque les projets sont menés entre école et musées, les degrés de participation entre les partenaires semblent plus équitablement distribués, dans le sens où le projet éducatif se coconstruit par l'ensemble des partenaires. En revanche, une seule étude traite d'une association entre écoles, familles et musées ; le degré d'investissement des partenaires y est plutôt du type volontariat (Auger et al., 2018).

10 Ces conclusions invitent à se pencher sur les contraintes et les obstacles associés à une participation plus engagée et soutenue des familles dans la mise en œuvre des projets partenariaux. Il semble central d'interroger les modes de participation des familles dans ces projets de recherche participative, surtout quand celles-ci sont issues de 
milieux fragilisés et/ou minoritaires. Nous analyserons ici le processus de constitution et de la dynamique d'un partenariat éducatif entre école, musées et familles, dans le contexte de cette recherche en cours à Montevideo (Uruguay).

\section{La mise en place de la recherche et de la démarche partenariale}

11 Les projets partenariaux comportent différentes étapes de mise en place (Depetris \& Eames, 2017). Le chemin que nous avons parcouru comprend 4 étapes: les deux premières concernent la construction du partenariat et le dessein du Projet Plurilingue et Interdisciplinaire (désormais PPI) ; la troisième - où nous sommes à l'heure actuelle correspond à la mise en place du $\mathrm{PPI}^{4}$; une quatrième étape portera sur l'extension du projet sur la base de l'analyse critique des étapes précédentes.

12 La préparation de l'entrée sur le terrain (Étape 1) a compris l'élaboration d'un prospectus présentant les trois chercheures universitaires impliquées et expliquant les buts de la recherche et de notre proposition, comme mobilisateur des motivations (Moore et al., 2018). Nous avons aussi procédé à un relevé exhaustif de tous les musées de Montevideo comportant des programmes éducatifs à destination de jeunes publics (février 2019), ce relevé étant fondamental pour informer et approcher tous les partenaires potentiels.

13 La sélection de l'école s'est effectuée sur deux critères : d'une part, le volontariat d'une équipe scolaire qui a toujours affiché une forte disponibilité pour accueillir des projets de recherche ; d'autre part, sa localisation, puisqu'il s'agit de la seule école d'éducation primaire et publique à la Ciudad Vieja, le quartier où se situe la plupart des musées, ce qui facilite la mobilité des participants, circonstance essentielle pour la viabilité de ce type de réseautage (Depetris \& Eames, 2017 ; Monroe et al., 2016). L'école constituant le nœud du réseautage partenarial, nous nous sommes appuyées sur la thématique nucléaire de son projet pour l'année scolaire 2019 - les jeux universels -, pour ensuite associer trois musées historiques et ethnographiques situés près de l'institution, ceci pour éviter des coûts associés au transport des partenaires et des participants du projet. Les trois musées contactés ont accepté de faire partie du partenariat: le MUMI (Museo de las Migraciones), le MAPI (Museo de Arte Precolombino e Indígena), et le musée historique de la ville (Museo Histórico Cabildo).

Pendant les mois de mars et avril 2019, les partenaires pressentis se sont réunis à trois reprises pour discuter du projet pédagogique et de son articulation à une recherche participative. Ces premières séances ont eu lieu à l'école pendant les rencontres hebdomadaires des professeurs. Le nombre élevé de personnes réunies (20), et le déséquilibre entre les cultures professionnelles représentées, avec une prédominance d'enseignants de l'école, se sont révélés peu productifs pour discuter ensemble de la construction d'un projet éducatif de l'école impliquant les musées et les familles. Ces déséquilibres ont par ailleurs constitué un obstacle de taille pour le développement d'une dynamique partenariale construite sur des expertises partagées pendant ces réunions, provoquant de fortes tensions à l'égard des rôles à jouer par chaque partenaire. L'expertise pédagogique des partenaires universitaires et des familles n'était pas nécessairement accueillie comme valide, tandis que familles et enseignants se sentaient peu légitimes au sein d'une collaboration de recherche. On voit ici se 
dérouler une perception différente, dans le sein du partenariat, de la recherche participative, en particulier entre une recherche "auprès " de participants ou bien «avec » eux (Litalien, Moore, \& Sabatier, 2012). Dès la fin de la troisième séance, un comité partenarial a été mis en place, composé d'un nombre moins important de participants, mais qui se déclaraient prêts à s'engager dans le développement et le pilotage du projet (participer régulièrement aux réunions, collaborer à la construction du matériel pédagogique et à la mise en place des activités didactiques, s'engager dans une démarche de recherche par le biais de la collecte de données et la participation à des discussions pédagogiques réflexives). Du côté de l'école, une seule enseignante a choisi de s'investir activement dans ce réseau, même si les autres enseignants ont manifesté leur accord pour mettre en place les activités dans leurs salles de classe, une fois celles-ci développées. Cette enseignante de l'école a joué un rôle fondamental pour que nous puissions présenter le projet aux familles des élèves.

Le tableau 1 présente le profil des 9 partenaires qui, au final, constituent le nœud de ce réseau partenarial et en assurent la cohérence et la continuité pour l'ensemble des autres acteurs engagés dans ce travail collaboratif. Nous y ajoutons la directrice de l'école (P2), qui, bien qu'absente physiquement à la plupart des réunions, a joué un rôle fondamental dans le fonctionnement de ce partenariat, surtout au moment de la mise en œuvre des activités du PPI et de la gestion des ressources scolaires.

Tableau 1 - Caractérisation des partenaires

\begin{tabular}{|c|c|c|c|c|}
\hline Code & Partenaire & Institution & Rôle & Profil linguistique et de mobilité \\
\hline P1 & scolaire & $\begin{array}{l}\text { école } \\
\text { primaire }\end{array}$ & enseignante & $\begin{array}{l}\text { Uruguayenne. Elle a vécu pendant } 10 \text { ans en } \\
\text { Galice. Elle est retournée en Uruguay il y a } 9 \\
\text { ans. }\end{array}$ \\
\hline P2 & scolaire & $\begin{array}{l}\text { école } \\
\text { primaire }\end{array}$ & directrice & $\begin{array}{l}\text { Uruguayenne. Elle déclare l'espagnol comme } \\
\text { sa langue maternelle. Elle a étudié le français, } \\
\text { l'anglais et l'italien, mais elle précise ne plus } \\
\text { les parler. Elle a vécu une partie de son } \\
\text { enfance (de } 1 \text { à } 5 \text { ans) en Argentine. }\end{array}$ \\
\hline P3 & muséal & $\begin{array}{l}\text { Musée } \\
\text { Historique } \\
\text { Cabildo }\end{array}$ & directrice & $\begin{array}{l}\text { Uruguayenne. Elle a toujours vécu en Uruguay. } \\
\text { Ses parents viennent de Galice et elle parlait le } \\
\text { galicien à la maison. Son rattachement à cette } \\
\text { langue est très fort, mais elle déclare } \\
\text { l'espagnol comme sa première langue. À } \\
\text { l'école, elle a étudié l'anglais et le français. }\end{array}$ \\
\hline P4 & muséal & musée MAPI & $\begin{array}{l}\text { médiatrice } \\
\text { (secteur } \\
\text { éducatif) }\end{array}$ & $\begin{array}{l}\text { Uruguayenne. Elle a toujours vécu en Uruguay } \\
\text { et ne parle et comprend que l'espagnol. }\end{array}$ \\
\hline P5 & muséal & $\begin{array}{l}\text { musée } \\
\text { MUMI }\end{array}$ & $\begin{array}{l}\text { médiatrice } \\
\text { (secteur } \\
\text { éducatif) }\end{array}$ & $\begin{array}{l}\text { Uruguayenne. Elle a toujours vécu en } \\
\text { Uruguay- L'espagnol est sa langue. Elle parle le } \\
\text { portugais et comprend l'anglais à l'écrit. }\end{array}$ \\
\hline
\end{tabular}




\begin{tabular}{|c|c|c|c|c|}
\hline P6 & familial & famille & mère & $\begin{array}{l}\text { Chilienne. Elle est récemment arrivée en } \\
\text { Uruguay. Elle a vécu en Argentine, au Brésil et } \\
\text { en Catalogne. L'espagnol est sa langue et elle } \\
\text { comprend le catalan. }\end{array}$ \\
\hline P7 & familial & famille & mère & $\begin{array}{l}\text { Cubaine. Elle est récemment arrivée en } \\
\text { Uruguay. Elle a vécu au Venezuela et au Brésil. } \\
\text { Elle parle le portugais. }\end{array}$ \\
\hline P8 & muséal & freelancer & $\begin{array}{l}\text { médiatrice } \\
\text { (secteur } \\
\text { éducatif) }\end{array}$ & $\begin{array}{l}\text { Uruguayenne. Elle a vécu en Colombie pendant } \\
\text { plusieurs années. Elle a travaillé avec des } \\
\text { populations minoritaires et migratoires dans } \\
\text { des ONG. Elle travaille comme freelancer dans } \\
\text { plusieurs musées. }\end{array}$ \\
\hline P9 & muséal & musée MAPI & $\begin{array}{l}\text { médiatrice } \\
\text { (secteur } \\
\text { éducatif) }\end{array}$ & $\begin{array}{l}\text { Uruguayenne. Elle a toujours vécu en Uruguay. } \\
\text { L'espagnol est sa langue maternelle et elle } \\
\text { comprend un peu de portugais et d'anglais. }\end{array}$ \\
\hline P10 & académique & université & $\begin{array}{l}\text { chercheuse } \\
\text { principale } \\
\text { (impliquée sur } \\
\text { le terrain) }\end{array}$ & $\begin{array}{l}\text { Portugaise (chercheuse principale). Elle vit en } \\
\text { Uruguay depuis } 12 \text { ans. Elle est enseignante et } \\
\text { doctorante. Elle a étudié le français, l'anglais, } \\
\text { l'espagnol, l'allemand, le latin et le russe. }\end{array}$ \\
\hline P11 & académique & université & $\begin{array}{l}\text { chercheuse } \\
\text { (co-direction de } \\
\text { la recherche) }\end{array}$ & $\begin{array}{l}\text { Portugaise. Elle vit au Portugal. Elle a étudié le } \\
\text { français, l'anglais, l'italien et l'allemand. Elle a } \\
\text { vécu en France. }\end{array}$ \\
\hline P12 & académique & université & $\begin{array}{l}\text { chercheuse } \\
\text { (co-direction de } \\
\text { la recherche) }\end{array}$ & $\begin{array}{l}\text { Française. Elle vit au Canada. Elle parle } \\
\text { français et anglais et s'intéresse à la } \\
\text { revitalisation des langues autochtones au } \\
\text { Canada. A vécu en France, en Angleterre, en } \\
\text { Suisse, au Canada, au Japon. }\end{array}$ \\
\hline
\end{tabular}

16 Le tableau 1 nous montre que ce partenariat est hétérogène sur plusieurs plans: les professions, les institutions d'appartenance et les cultures professionnelles des acteurs sont différentes. Le projet intègre par ailleurs des personnes présentant des parcours de vie diversifiés avec des trajectoires plurilingues et des expériences de mobilité variées. Cette hétérogénéité s'est révélée assez utile à l'heure de la conceptualisation des activités plurilingues du projet, lesquelles sont le résultat du partage des expériences, des savoirs et des savoir-faire des membres du partenariat. Ainsi, le PPI comprend un ensemble d'activités menées dans une perspective dialogique qui englobe, en continuum et en interaction permanente, tous les partenaires: des ateliers dans l'école dynamisés par plusieurs acteurs éducatifs (enseignants, chercheurs, familles, animateurs des musées), des visites guidées et interactives aux musées, des ateliers dans les musées, des promenades par le paysage linguistique (visuel et sonore) du quartier de l'école, la création de dispositifs muséologiques par les enfants. 


\section{La mise en place d'un partenariat école-musées- familles : quels enjeux?}

17 Le corpus d'étude, sur lequel s'appuie cette contribution, comprend trois types de données discursives recueillies pendant les deux premières étapes du projet (initiation et constitution du partenariat) et au début de la troisième (mise en place du PPI) : le carnet de bord de la chercheuse principale ${ }^{5}$, l'enregistrement des interactions asynchrones, multilatérales et bilatérales, entre les membres du partenariat (courrier électronique et Whatsapp), la transcription de l'enregistrement d'une rencontre du partenariat portant spécifiquement sur les motivations des partenaires, les dynamiques du réseautage et les possibilités et perspectives de participation dans une recherche académique.

Pour ces analyses, nous avons utilisé un logiciel d'analyse qualitative (webQDA) qui permet d'établir, individuellement ou collectivement, de manière synchrone ou asynchrone, un système catégoriel pour filtrer et organiser les données discursives en unités signifiantes pour les partenaires de la recherche. L'analyse nous a ainsi permis de noter l'existence de catégories avec une forte occurrence d'unités de contenu, ces catégories étant en rapport avec : (i) les rôles assumés par les partenaires, (ii) les défis posés par le partenariat et (iii) les possibilités de mise en marche d'une recherche participative.

\section{Les rôles des partenaires}

\section{Un rôle endossé de facilitatrice pour la chercheure sur le terrain (P10)}

19 La construction d'un partenariat éducatif part souvent de la conjugaison d'un besoin individuel et d'une problématique sociale identifiée par un individu qui s'intéresse simultanément aux questions éducatives et communautaires, et qui prend l'initiative de créer un partenariat pour les aborder. Après la constitution du partenariat, cette personne, désignée comme la facilitatrice, conserve souvent des responsabilités de coordination et de gestion du réseautage (Monroe et al., 2016).

Dans notre étude, la chercheuse principale (P10) a joué ce rôle dès le début du projet, en particulier au niveau de la coordination générale du réseau, de la communication, de la gestion des tensions, de l'opérationnalisation du PPI et du maintien de la motivation des partenaires. Ce rôle concerne tout particulièrement :

- la coordination des rencontres,

Inicialmente, tínhamos marcado esta reunião para dia 9, no MUMI, mas, como havia poucas confirmações, decidi adiá-la para esta sexta-feira. » (CR/ $16 / 08 / 2019)^{6}$

- la préparation des rencontres et du matériel du partenariat,

Durante esta semana, fui preparando diversos materiais para a reunião. (CR/ $06 / 09 / 2019)^{7}$

- la communication, 
Logo pela manhã comecei a ligar aos pais que não me tinham confirmado a receção da mensagem. (CR/07/06/2019) ${ }^{8}$

- l'opérationnalisation du PPI et la mobilisation des partenaires,

Enviei um email à parceria com um resumo da reunião. Era necessário começar a definir vários assuntos e mobilizar os parceiros que têm tido uma presença mais tímida na construção de algumas atividades. (CR/19/08/2019) ${ }^{9}$

- la gestion des tensions,

Vou tentar reunir-me com ela na quinta-feira [...] e tentar convencer, mas sem impor, a ideia da parceria, mais no sentido de que em rede conseguimos fazer mais coisas [...] Tenho de gerir isto com pinças. $(\mathrm{CR} / 09 / 03 / 2019)^{10}$

21 Ces tâches appellent des compétences diversifiées de la part de P10, en particulier des compétences stratégiques et de négociation qui transforment la démarche partenariale en une tâche exigeante et, dans certaines situations, assez ardue :

"Começa a ser pesado, duro mesmo, ter de lidar com esta situação de liderança da diretora $\left[\right.$ da escola]... » $(\mathrm{CR} / 08 / 05 / 2019)^{11}$.

Le rôle de facilitatrice de P10 est reconnu par tous les partenaires comme essentiel au bon fonctionnement du projet pédagogique collaboratif ( $y$ que tú estás haciendo un trabajo como de ir enlazando todo $»^{12}$ - P8/RR/06/09/2019). Les participants considèrent même que ce rôle est le plus difficile à endosser dans une dynamique partenariale («lo organizativo es lo más difícil del trabajo en red $»^{13}$ - P1/RR/06/09/2019). On note par ailleurs la stabilité dans ce rôle auto et hétéro-assigné au sein du réseau partenarial, ce qui n'est pas nécessairement le cas pour ceux joués par les autres partenaires, en particulier les enseignants et les parents, comme nous allons pouvoir le voir ci-après.

\section{Des rôles partagés entre partenaires}

Le partenariat de notre recherche se caractérise par le jumelage de cultures professionnelles et éducatives et de terrains d'action très diversifiés : "las dimensiones del trabajo de cada uno / los accionares de cada uno /son muy diferentes. ${ }^{14}$ - P1/RR/ 06/09/2019) (cf. tableau 1). La dynamique partenariale se caractérise en effet souvent par la fluidité et la flexibilité des rôles assumés par des partenaires (Epstein, 2002), dont l'engagement dépend souvent du contexte et de la culture de travail ou institutionnelle de laquelle ils proviennent.

Cette hétérogénéité reconnue par les sujets conduit à des modalités de participation très différentes et des rôles clairement identifiables, notamment au sein de l'institution scolaire. Ainsi, nous avons pu vérifier que les musées sont plus participatifs et engagés dans la mise sur pied du projet que les autres partenaires (école, familles, chercheurs). Nous avons noté en particulier que travailler avec des musées, c'est aussi partager des idées avec toute l'équipe éducative muséale qui reste, dans la plupart des cas, dans les coulisses du partenariat : "Para P4, é essencial a contribuição de toda a equipa educativa » ${ }^{15}$ $(\mathrm{CR} / 22 / 05 / 2019)$. Ce sont aussi les musées qui ont le plus impulsé le développement de ressources permettant la mise en place des activités-clés du projet pédagogique : «A P3 deu logo uma ideia genial de uma exposição que ela tinha visto em Buenos Aires com mapas sonoros ${ }^{16}(\mathrm{CR} / 22 / 05 / 2019)$. 

la logistique des activités, tout en identifiant des possibilités et d'éventuelles contraintes à leur mise en place : «P6 e P7 estavam atentas a pormenores como a gestão do tempo $»^{17}(\mathrm{CR} / 22 / 06 / 2019)$. De ce fait, pour que les parents (dans la situation étudiée, des mères) participent de manière engagée, il était nécessaire de les (re)contacter individuellement, par des messages Whatsapp, en leur proposant de partager des responsabilités de conceptualisation et de dynamisation de quelques activités-clés qui doivent se dérouler, par exemple, lors des visites dans les musées (comme participer à la prise de photos ethnographiques ou le recueil des sons de la ville avec les enfants). Leur entrée plus tardive dans le réseau semble avoir restreint leur participation active dans la conceptualisation du projet et des activités et ressources pédagogiques. joué un rôle fondamental de gardienne (gatekeeper) de la recherche et du projet chez les enseignants ${ }^{18}$ en accomplissant aussi d'autres tâches telles que l'explicitation du projet pédagogique aux familles (avec P1, qui a joué ce même rôle) et le partage d'informations concernant le PPI avec d'autres éventuels collaborateurs (« Vos hablá con los museos y dejá las maestras para mí $»^{19}-\mathrm{CR} / 27 / 02 / 2019$; " Os do CdF tinham estado pela escola, ela comentou-lhes sobre o projeto e passou-lhes o meu contacto, pois eles tinham-se mostrado interessados $\left.»^{20}-\mathrm{CR} / 12 / 08 / 2019\right)$. D'une façon plus large, nous remarquons que P2 a joué un rôle essentiel dans l'opérationnalisation de ce projet éducatif multi-sites, et ceci à plusieurs niveaux. P2 investit la responsabilité de tâches aussi diverses que la relecture critique du matériel de diffusion produit par le partenariat ainsi que la relecture du guide d'activités du PPI, la programmation des espaces et des horaires des activités à implémenter à l'école, et la mise à disposition du matériel didactique et des autres ressources nécessaires comme, par exemple, des photocopieuses.

Au final, si les musées semblent plus engagés dans le développement des ressources pédagogiques et dans la mise en place des activités didactiques, ce sont bien les partenaires issus de l'institution scolaire qui sont le nœud spatial et le ciment du partenariat, en assurant l'engagement des élèves et des familles, et la continuité du projet.

\section{Les défis d'une posture de chercheur-acteur dans une démarche partenariale participante}

La conceptualisation, la planification et la mise en œuvre d'un partenariat comportent, nous l'avons vu, de nombreux défis et demandent de la part de tous les acteurs impliqués la prise en charge de plusieurs types de responsabilités, ainsi que des compétences interpersonnelles et stratégiques. Nous nous intéressons ici plus particulièrement aux défis auxquels engage la posture de chercheur-acteur dans la démarche partenariale participante de $\mathrm{P} 10$, tout particulièrement au moment de la mise en marche du partenariat (étape 2). Cette étape s'est particulièrement marquée par de nombreux moments de risque qui ont mobilisé ses compétences interpersonnelles et stratégiques de facilitatrice, en particulier au niveau de la gestion des tensions.

Les analyses ont permis de mettre en avant deux aspects qui caractérisent ces moments de risque pour le chercheur-acteur. Un premier se rattache aux attentes initiales de la part de la directrice de l'école (P2) par rapport au projet éducatif plurilingue et 
interculturel, qu'elle pensait pouvoir fusionner avec le projet pédagogique de l'école, centré cette année-là sur les jeux universels. De l'avis de P2, les rencontres hebdomadaires des enseignants de l'école avec les autres partenaires étaient, ainsi, la meilleure procédure pour travailler ensemble. Pourtant, après deux ou trois de ces rencontres, P10 note, dans ses observations et ses actions de terrain, combien cette décision influe sur l'organisation de cet espace fortement marqué par la culture scolaire (" O clima marca muito : este é o espaço dos professores. »" - CR/07/05/2019). Aussi elle considère que cet espace de réunion était très (trop) peuplé (avec plus de 20 personnes) et, de la sorte, susceptible de réduire le potentiel créatif d'un projet plurilingue partenarial ( as salas eram muito absorventes, os professores acabam por ter quase todo o "tempo de antena" e não são um espaço nada prático para a criação de um projeto plurilingue $\left.»^{22}-\mathrm{CR} / 22 / 05 / 2019\right)$. La mise en place du partenariat appelle flexibilité et adaptation de la part de P2, de la part des autres partenaires, et tout particulièrement de la part de P10, dont le rôle ne se borne pas à observer les participants: P10 y joue une importante fonction d'impulsion des actions, de coordination et de stimulation des partenaires impliqués.

Un deuxième exemple montre comment P10 adapte la forme de la participation des partenaires en changeant les codes préalablement co-établis des réunions du groupe, en particulier par P2. Le modèle très collégial, ouvert à tous, fondé sur la mise en commun d'expertises diversement situées qui était celui des premières réunions, parce qu'il ne réussit pas à engager la participation, est remplacé en cours de route par une redistribution des rôles des participants. Se constitue alors, sous l'impulsion de P10, un comité de pilotage qui assume cette responsabilité, jusque-là surtout endossée par P2: ( A P4 corroborou [...] que achava que estávamos "colgadas" na reunião, que não havia abertura $\left.»^{23}-\mathrm{CR} / 09 / 05 / 2019\right)$.

Une autre situation problématique est en rapport avec la nécessité ressentie par P10, dans son rôle de chercheuse principale de l'étude : motiver constamment les membres du réseautage afin d'éviter les abandons en cours de route, tout en cherchant les stratégies et les modalités les plus adéquates pour dépasser les contraintes de toute sorte perçues par les partenaires: «A P4 comentou que está a fazer de tudo para que a atividade vá avante, mas desabafou que, até ontem, pensava que o MAPI teria de desistir da

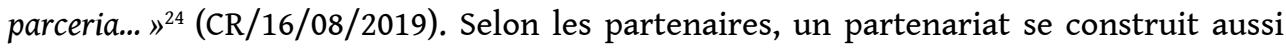
selon un processus d'entonnoir (embudo), c'est-à-dire, par un moment, souvent initial, d'abandon de certains partenaires: «después comienza como el embudo / como a decantar $\|^{25}$ (P1/RR/06/09/2019). Par exemple, la gestion du temps (comment faire coïncider les contraintes horaires des différents membres) s'est constituée comme un nœud caractéristique limitant la participation des partenaires ( $A$ grande maioria dos familiares apenas podia da parte da manhã, o que tornaria difícil a participação da P1 $»^{26}-\mathrm{CR} /$ 06/06/2019). Si la mise en place de modalités alternatives de communication, comme le courrier électronique ou les messages Whatsapp, a permis de lever certaines barrières, il reste que les pratiques linguistiques et culturelles et les savoirs des familles ont difficilement pu être investis directement, pour le moment, au sein du projet. On observe toutefois que les activités pédagogiques développées collaborativement donnent voix aux communautés locales ${ }^{27}$, au musée et dans la rue, interrogeant leur(s) histoire(s), leurs objets et leurs pratiques.

Ces défis qu'il faut gérer en temps réel et qui engagent le chercheur à jouer de différents équilibres dans la posture de chercheur-acteur, définissent au final le 
caractère de la participation. En effet, ces moments de doute dans la construction partenariale, ainsi que les glissements dans les rôles et les postures participatives, sont de nature à favoriser la réflexivité et le dialogue et, partant, d'interroger le processus participatif dans la recherche.

\section{Le partenariat comme déclencheur de la participation en recherche}

Un espace partenarial où un ensemble d'acteurs sociaux partage des expériences et des ressources pour construire un projet plurilingue favorise, à notre avis, la recherche participative. Autrement dit, ces acteurs sociaux s'y configurent comme des sources de co-production de connaissances (Gillet \& Tremblay, 2017). En nous appuyant sur l'analyse des voix plurielles des partenaires, nous cherchons à comprendre les possibilités de mise en place d'une recherche participative et les enjeux qu'implique une telle démarche dans notre contexte de recherche, afin de tracer le meilleur chemin possible.

Mobiliser une diversité d'expertises autour d'un projet commun (ici autour du plurilinguisme) pour mieux le comprendre et le mener à bien, se situe comme l'enjeu central de la mise en œuvre du partenariat. Pouvoir développer une culture commune de recherche dans l'intervention, un savoir-faire méthodologique (" ¿Y cómo serían esas instancias? ¿Serían como estas? ¿Cómo sería la metodología? " $\left.{ }^{28}-\mathrm{P} 8 / \mathrm{RR} / 06 / 09 / 2019\right)$, pouvoir répartir les rôles de chacun dans la co-construction des savoirs (" ¿Qué aportar a la investigación ? " ${ }^{29}$-P4/RR/06/09/2019), constituent des nœuds de questionnements récurrents à cet égard. Par exemple, l'observation en tant que modalité de recherche participative qui donne accès à une compréhension plus approfondie et partagée des activités développées par le partenariat (comme l'observation des extraits des enregistrements vidéos de ce que font les enfants) est considérée par les partenaires comme une activité complexe, dont ils considèrent qu'ils ne maitrisent ni les enjeux ni les techniques ("Con qué claves tendríamos que mirar y observar qué cosas en esas instancias // no me queda de todo claro $»^{30}$ - P8/RR/06/09/2019), ce qui confirme l'importance de trouver des modalités de "médiation entre les cultures de pratiques et celles de la recherche » (Giglio, $2016: 46)$. En revanche, la participation des différents membres à la collecte des données (comme l'enregistrement photographique des activités du projet ou l'enregistrement audiovisuel du feedback des participants après leur mise en place), s'est avérée plus aisée pour les différents membres du réseau qui se sont ainsi sentis vraiment inclus dans le processus de recherche collaborative : «P8 até se prontificou a fazer o levantamento fotográfico das atividades... $»^{31}(\mathrm{CR} / 21 / 03 / 2019)$ / « A mí me parece bien / lo que dijo P8/sacar ahí en el momento alguna opinión... » ${ }^{32}$ (P4/RR/06/09/2019). Il reste plus difficile pour les différents partenaires de comprendre la valeur des savoirs et des langues des familles (y compris et peut-être surtout de la part de celles-ci) dans ce projet de recherche participative qui pourtant s'organise autour du développement d'activités et de ressources plurilingues par/pour le plurilinguisme. Les parents sont restées jusqu'ici plus en retrait, une tendance que nous espérons voir s'inverser dans les prochaines étapes du travail collaboratif.

La mise en place d'une recherche participative est vécue comme le résultat naturel de ce projet conçu à partir d'une démarche partenariale ( «A mí/ sí/ porque es parte // es un poco del por qué hicimos esto / ¿no? / Es lo que salió de todo esto ¿no?»»33-P4/RR/ 
06/09/2019). Ce sont en effet les malentendus et la mise en évidence des écarts des attentes, des représentations et des pratiques qui fondent un processus qui fait appel à l'engagement, à la réflexivité et à de nouveaux apprentissages («Es todo un aprendizaje $\left.\|^{34}-\mathrm{P} 1 / \mathrm{RR} / 06 / 09 / 2019\right)$, aspects qui sont au cœur de ce type de recherche (Gillet \& Tremblay, 2017). Ce sont bien dans ces interstices que se construit la collaboration.

\section{Conclusion}

Nous avons dans cette contribution cherché à mettre en avant certaines dynamiques de la collaboration au moment de l'établissement et de la mise en place d'un projet éducatif impliquant un partenariat visant à engager école, familles et institutions communautaires (comme ici les musées) autour de la valorisation du plurilinguisme de jeunes élèves de l'école primaire en Uruguay. Nous y avons retracé différentes étapes de la mise en place d'un tel projet pour discuter ensuite comment se construit la participation des partenaires, ainsi que le rôle ambigu du chercheur-acteur dans ces configurations partenariales multi-situées. Nous avons cherché à montrer combien une telle démarche de recherche, qui se construit comme variable, complexe et dynamique (Blanchet \& Chardenet, 2011), est reliée à des questionnements fondamentaux sur la responsabilité sociale de la recherche et sur l'éthique dans le processus de construction de la connaissance (Anadon, 2013). Ainsi, la recherche participative doit-elle s'accompagner du partage de volontés et de valeurs, de la complémentarité d'expertises et de compétences, et de démarches adaptées aux contextes d'appartenance et d'action des partenaires de la recherche, tout autant qu'à l'écologie locale qui marque leur domaine d'action.

En éclairant certains enjeux qui entourent les conditions de mises en place de recherches qui impliquent les enseignants, les familles, les élèves et les intervenants muséaux comme coparticipants dans des expériences éducatives où les questionnements sont impulsés, nous espérons contribuer, à notre mesure, à un questionnement qui demeure central dans la recherche actuelle en DLP. C'est en effet la mise en réseau des professionnels de l'éducation (scolaire et muséale) et des familles qui permet, pour l'enfant, d'investir la continuité des apprentissages entre les différents pôles qui constituent son univers quotidien (Bottoms et al., 2017).

Quand ce partenariat associe aussi les institutions muséales dans les projets d'apprentissage, on voit se démultiplier l'expérience de l'altérité. Nos analyses soulignent la valeur de ces espaces de collaboration qui favorisent l'expression des acteurs et le partage de leurs expériences, l'innovation pédagogique, le développement de la professionnalité par/dans la recherche, l'action et la formation (Giglio, 2016) mais aussi la difficulté de leur mise en place. En ce sens, le partenariat comme déclencheur de la recherche participative inscrit la démarche comme un «incubateur de ressources » (Sperano, Roberge, Bénech, Trgalova, \& Andruchow, 2019). On espère dans la suite de nos recherches pouvoir montrer que ce partenariat, qui s'inscrit dans un paradigme de la diversité, a des effets bénéfiques pour l'ensemble des apprenants et des partenaires éducatifs (Beacco et al., 2016). 


\section{BIBLIOGRAPHIE}

Anadon, M. (2013). Recherche participative. Dans I. Casillo, R. Barbier, L. Blondiaux, F. Chateauraynaud, J.-M. Fourniau, R. Lefevre, D. Salles (eds.), Dictionnaire critique et interdisciplinaire de la participation. Paris : GIS Démocratie et Participation. Disponible à : http://www.dicopart.fr/ it/dico/recherche-participative.

Auger, N., Azaoui, B., Houée, C., \& Miquel, F. (2018). Le projet européen Romtels (Roma translanguaging enquiry learning spaces). Recherches en didactique des langues et des cultures, 15(3), 1-21. Disponible à : https://doi.org/10.4000/rdlc.3321.

Bautista Garcia-Vera, A., Limón Mendizábal, M. R., Oñate, P., De La Rasilla, G., \& Rostand Quijada, C. (2016). Funciones de la fotografía en las relaciones interculturales entre familias inmigrantes. Revista Complutense de Educación, 27(1), 75-93. Disponible à : https://doi.org/10.5209/rev_RCED. 2016.v27.n1.45115.

Beacco, J.-C., Byram, M., Cavalli, M., Coste, D., Cuenat, M. E., Goullier, F., \& Panthier, J. (2016). Guide pour le développement et la mise en œuvre de curriculums pour une éducation plurilingue et interculturelle. Strasbourg : Conseil de l'Europe. Disponible à : https://rm.coe.int/16806ae64a.

Beaumont, S. \& Moore, D. (2020). La recherche-action-formation comme nœud de la professionnalité enseignante? Le cas des enseignants de l'éducation nationale française en Amérique du Nord. Dans Actes du IV colloque international, Former et développer l'intelligence professionnelle. Sherbrooke, 94-103.

Blanchet, P., \& Chardenet, P. (2011). Guide pour la recherche en didactique des langues et des cultures. Approches contextualisées. Paris : Éditions des archives contemporaines et Agence Universitaire de la Francophonie. Disponible à: http://www.bibliotheque.auf.org/doc_num.php?explnum_id=819.

Bottoms, S. A. I., Ciechanowski, K., Jones, K., de la Hoz, J., \& Fonseca, A. L. (2017). Leveraging the community context of Family Math and Science Nights to develop culturally responsive teaching practices. Teaching and Teacher Education, 61, 1-15. Disponible à : https://doi.org/10.1016/j.tate. 2016.09.006.

Charalampopoulou, C. (2013). L'éducation interculturelle par le biais des musées : revue de littérature. Dans Congrès de l'Actualité de la Recherche en Éducation et Formation. Montpellier. Disponible à : https://hal.archives-ouvertes.fr/hal-01903145.

Clerc, S. (2015). Construire une éducation plurilingue et interculturelle avec les parents : enjeux et modalités. Babylonia, 2(15), 34-37. Disponible à : https://www.ecml.at/Portals/1/resources/ Articles.

Depetris, T. \& Eames, C. (2017). A Collaborative Community Education Model : Developing Effective School-Community Partnerships. Australian Journal of Environmental Education, 33(3), 171188.

Desgagné, S. (1997). Le concept de recherche collaborative : l'idée d'un rapprochement entre chercheurs universitaires et praticiens enseignants. Revue Des Sciences de l'éducation, 23(2), 371393.

Epstein, J. (2002). School, Family, and Community Partnerships : Caring for the Children We Share. In Epstein, J. L., Sanders, M. G., Simon, B. S., Salinas, K. C., Jansorn, N. R., \& Van Voorhis, F. L. (Eds.). School, family, and community partnerships : Your handbook for action. Thousand Oaks, CA : Corwin, p. 7-29. 
Flückiger, B., Diamond, P. \& Jones, W. (2012). Yarning space : Leading literacy learning through family-school partnerships. Australasian Journal of Early Childwood, 37(3), 53-59.

Giglio, M. (2016). Créativité et professionnalité de l'enseignant : une démarche de rechercheinnovation-formation. Formation et Profession, 24(2), 45-55. https://doi.org/10.18162/fp.2016.349

Gillet, A., \& Tremblay, D.-G. (2017). Les recherches partenariales et collaboratives. Québec : Presses de l’Université du Québec.

Ishimaru, A. M., Barajas-López, F., \& Bang, M. (2015). Centering family knowledge to develop children's empowered mathematics identities. Journal of Family Diversity in Education, 1(4), 1-21. Disponible à : http://familydiversityeducation.org/index.php/fdec.

Li, G., Anderson, J., Carr, W. \& Hare, J. (2018). Supporting teachers with diversity-plus competencies for working with culturally, linguistically, and racially diverse students, families and communities. Journal of Family Diversity Education, hors série 3(1), 1-91.

Litalien, R., Moore, D. \& Sabatier, C. (2012). Ethnographie de la classe, pratiques plurielles et réflexivité : pour une écologie de la diversité en contexte francophone en Colombie-Britannique. Revue canadienne de l'Éducation, 35(2), 192-211.

Milutinović, J., \& Gajić, O. (2010). Intercultural dialogue in the museum context. US-China Educational Review, 7(7), 30-42. Disponible à : https://files.eric.ed.gov/fulltext/ED511336.pdf.

Monroe, M. C., Ballard, H. L., Oxarart, A., Sturtevant, V. E., Jakes, P. J., \& Evans, E. R. (2016). Agencies, educators, communities and wildfire : partnerships to enhance environmental education for youth. Environmental Education Research, 22(8), 1098-1114.

Moore, D. (sous presse). Connecting educators, families and communities through PASTEL (Plurilingualism, Art, Science, Technology and Literacies) approaches in and around French immersion. Dans Li, G. \& Anderson, J. (Eds.). Teacher Education in a Superdiverse World. Londres, Routledge.

Moore, D., Hoskyn, M., \& Mayo, J. K. (2018). Thinking Language Awareness at a Science Centre : Ipads, science, and early literacy development with multilingual kindergarten children in Canada. International Journal of Bias, Identity and Diversities in Education, 3(1), 40-63.

Santos, M., Araújo e Sá, M. H., \& Simões, A. R. (2012). Interculturality and intercultural education - Representations and practices of a group of educational partners. L1 Educational Studies in Language and Literature, hors-série 12, 1-21.

Simonin, M.-C., \& Thamin, N. (2018). Recherche collaborative à l'école maternelle et socialisation plurilingue. Revue Diversité, La Recherche En Éducation. Vers de Nouvelles Interfaces, 192, 131-136.

Sperano, I., Roberge, J., Bénech, P., Trgalova, J., \& Andruchow, R. (2019). Exploring New Usages of Journey Maps : Introducing the Pedagogical and the Project Planning Journey Maps. Advances in Intelligent Systems and Computing, 824, 964-982. https://doi.org/10.1007/978-3-319-96071-5_99

Taylor, L. K., Bernhard, J. K., Garg, S., \& Cummins, J. (2008). Building on Students' Family-Based Cultural and Linguistic Capital through a Multiliteracies Curriculum. Journal of Early Childhood Literacy, 8(3), 269-295.

Walton, J., Paradies, Y., \& Mansouri, F. (2016). Towards reflexive ethnicity : Museums as sites of intercultural encounter. British Educational Research Journal, 42(5), 871-889. 


\section{NOTES}

1. La première auteure de cet article est la chercheuse principale, celle qui a mené l'étude de terrain. Les autres deux co-auteures sont les directrices de la recherche.

2. Il y a trois types d'écoles primaires : les écoles Tiempo Completo (temps complet, 7h30/jour), les écoles Tiempo Extendido (6h/jour) et les écoles Comunes (4h/jour).

3. Epstein (2002) identifie 6 types de participation par degrés croissants d'investissement : 1) rôle parental ; 2) communication ; 3) volontariat ; 4) apprentissage à la maison ; 5) prise de décision ; 6) collaboration avec la communauté.

4. Différents partenaires sont entrés à des moments différents des étapes de mise en place de ce réseautage.

5. 40 entrées ont été objet d'analyse (depuis l'entrée sur le terrain de la chercheuse principale, en février 2019, jusqu'à la première rencontre réflexive du partenariat réalisée au début de septembre 2019).

6. « Au départ, nous avions prévu cette réunion pour le 9 , au MUMI, mais, comme il y avait peu de confirmations, j'ai décidé de la reporter à ce vendredi ».

7. «Au cours de cette semaine, j'ai préparé divers documents pour la réunion ».

8. « Tôt le matin, j’ai commencé à appeler les parents qui n'avaient pas confirmé la réception du message ».

9. «J'ai envoyé un courriel au partenariat avec un résumé de la réunion. Il a fallu commencer à définir différentes problématiques et à mobiliser les partenaires qui ont eu une présence plus timide dans la construction de certaines activités ».

10. « Je vais essayer de la rencontrer jeudi [...] et essayer de la convaincre, mais sans imposer, sur l'idée du partenariat [...] plus dans le sens que dans un réseau on peut faire plus de choses [...] je dois gérer ça avec une pince à épiler».

11. « Ça commence à être lourd, vraiment dur, d'avoir à faire face à cette situation de leadership du directeur [de l'école] ... »

12. "... et que vous faites un travail comme de tout relier".

13. «L'organisation est le plus difficile dans un travail en réseau ».

14. «Les dimensions de travail de chacun/ les actions de chacun/ sont très différentes ».

15. « Pour P4, la contribution de toute l'équipe pédagogique est essentielle ».

16. « $\mathrm{P} 3$ a immédiatement donné une excellente idée d'une exposition qu'elle avait vue à Buenos Aires avec des cartes sonores ».

17. « P6 et P7 étaient attentives aux détails tels que la gestion du temps ».

18. On notera que $\mathrm{P} 11$ et $\mathrm{P} 12$ jouent un rôle similaire auprès de $\mathrm{P} 10$. Nous ne l'analysons pas ici.

19. "Vous vous occupez des musées et vous me laissez les enseignantes».

20. «Ceux du CdF étaient allés à l'école, elle a commenté le projet et transmis mon contact, car ils avaient montré de l'intérêt ».

21. «L'ambiance marque beaucoup : c'est l'espace des professeurs".

22. «Les salles étaient très absorbantes, les professeurs ont fini par avoir presque tout le « temps d'antenne » et ce n'était pas du tout un espace adéquat pour créer un projet plurilingue ».

23. « $\mathrm{P} 4$ a corroboré [...] le fait qu'elle pensait que nous étions « coincés » à la réunion, qu'il n’y avait pas d'ouverture ».

24. «P4 a commenté qu'elle faisait tout pour faire avancer l'activité, mais elle a confié que jusqu'à hier, elle pensait que le MAPI devrait abandonner le partenariat ».

25. «Puis cela commence comme l'entonnoir / c'est comme décanter ».

26. «La grande majorité des membres de la famille ne pouvait que le matin, ce qui rendrait difficile la participation de P1». 
27. Par le biais, par exemple, de l'étude des sons de la ville effectuée lors d'une sortie au musée des enfants (une des activités plurilingues développées par l'équipe partenariale, activités qui ne sont pas explorées dans le cadre de cet article).

28. «Et à quoi ressembleraient ces rencontres? Seraient-elles comme celles-ci ? À quoi ressemblerait la méthodologie?».

29. «Quoi amener à la recherche?»

30. «Avec quelles clés nous devrions regarder et observer quelles choses dans ces cas? Ce n'est pas clair pour moi ».

31. «P8 a même proposé de faire un relevé photographique des activités ... ».

32. «Cela me semble bien/ ce que P8 a dit / avoir un feedback là-bas tout de suite... ».

33. «Pour moi / oui / parce que cela fait partie // c'est un peu pourquoi nous avons fait ça / non ? / C'est ce qui est sorti de tout ça non?».

34. « C'est tout un apprentissage ».

\section{RÉSUMÉS}

La contribution porte sur une recherche en cours dont l'objectif est de comprendre comment se construit un partenariat pédagogique et de recherche impliquant un travail collaboratif entre une école, des familles et plusieurs musées de la ville, autour du développement de scénarios didactiques pour/ par le plurilinguisme pour des enfants du primaire, à Montevideo (Uruguay). L'enjeu est d'éclairer certaines conditions d'élaboration d'un tel partenariat impliquant des enseignants, des praticiens de l'éducation muséale, des chercheurs en didactique des langues et des parents d'élèves. Par le biais d'une approche (auto)-ethnographique, nous chercherons à comprendre : (i) comment ce partenariat éducatif est envisagé par les différents partenaires - ses enjeux et ses contraintes pour chacun; (ii) leurs rôles dans l'éclairage et la compréhension d'une recherche visant une innovation pédagogique autour du plurilinguisme. Nous explorerons en particulier quelques implications pour le chercheur-acteur, ainsi que les transformations qu'elles supposent, tant au niveau des pratiques professionnelles que des pratiques et de la posture de recherche. La contribution pose ainsi quelques jalons pour mieux comprendre les enjeux, les savoirs et les méthodes des recherches collaboratives en didactique des langues et du plurilinguisme, ainsi que leur potentiel pour la transformation des pratiques (de recherche et en éducation).

Este texto apresenta uma investigação em curso cujo objetivo é a compreensão de como se constrói uma parceria educativa e de investigação entre uma escola, famílias e diversos museus da cidade, implicados no desenvolvimento de cenários didáticos para e pelo plurilinguismo destinados a alunos de uma escola primária em Montevideu, no Uruguai. Pretende-se refletir sobre as condições de formação de uma tal parceria implicando professores, mediadores de museus, famílias de alunos e investigadores em didática de línguas. Inserida num paradigma (auto)-etnográfico, a investigação visa compreender : 1) como é que este partenariado educativo é compreendido pelos sujeitos (suas potencialidades e constrangimentos) ;2) quais são os papéis dos parceiros numa investigação visando a inovação pedagógica em torno do plurilinguismo. Exploramos, ainda, algumas implicações para o investigador-ator, bem como as transformações que elas acarretam tanto ao nível das práticas profissionais como das práticas e da postura da investigação. $O$ estudo permite identificar alguns elementos fundamentais para aprofundar o 
conhecimento sobre as possibilidades, saberes e abordagens de investigação colaborativa em didática das línguas e do plurilinguismo, bem com o seu potencial para a transformação das práticas (de investigação e de educação).

\section{INDEX}

Mots-clés : partenariat école-musée-famille, plurilinguisme, recherche participative

Keywords : partnerships schools-museums-families, plurilingualism, participative research

\section{AUTEURS}

\section{RAQUEL CARINHAS}

Universidade de Aveiro, CIDTFF, Universidad de la República, Camões, I.P Doctorante du Programme Doctoral en Éducation de l'Université d'Aveiro, Portugal. Chercheuse dans le domaine de la didactique des langues et du plurilinguisme. Elle s'intéresse actuellement à des questions liées aux partenariats éducatifs entre école, familles et musées dans une visée d'éducation plurilingue et interculturelle. Lectrice de portugais du Camões - Institut de la Coopération et de la Langue à l'Université de la République à l’Uruguay. raquelcarinhas[at]ua.pt

\section{MARIA HELENA ARAÚJO E SÁ}

Universidade de Aveiro, CIDTFF

Professeure de Didactique des Langues au Département d'Éducation et Psychologie de l'Université d'Aveiro, Portugal. Elle dirige actuellement le Centre de Recherche en Didactique et Technologie dans la Formation des Formateurs (CIDTFF) et est coresponsable du L@LE, laboratoire qui travaille dans le domaine de la Didactique du Plurilinguisme et de l'Interculturalité. Elle est responsable du Doctorat en Éducation de son université. helenasa[at]ua.pt

\section{DANIÈLE MOORE}

Simon Fraser University, CRECHE - PluriLCo et DILTEC, Paris 3 Sorbonne Nouvelle Professeure à la Faculté d'Éducation de l'université Simon Fraser à Vancouver au Canada, et Directrice de recherche (HDR) à la Sorbonne Nouvelle - Paris 3 en France, ses recherches couvrent les domaines de la sociolinguistique et de la didactique des langues et $\mathrm{du}$ plurilinguisme. Ses travaux récents investissent des recherches collaboratives portant sur le développement de pratiques éducatives innovantes autour du plurilinguisme et de la revitalisation des langues autochtones, ces recherches impliquant écoles, familles, centres communautaires et musées. dmoore[at]sfu.ca 